Kong. Res. J. 1(1) : 27-28, 2014

Kongunadu Arts and Science College, Coimbatore

\title{
B-CHROMATIC NUMBER OF CENTRAL GRAPH OF LADDER GRAPH AND COMPLETE GRAPH
}

\author{
Vijayalakshmi, D*. \\ Department of Mathematics, Kongunadu Arts and Science College, Coimbatore - 641029 \\ *Email: vijikasc@gmail.com
}

\begin{abstract}
In this paper, we discuss about the b-colouring andb-chromatic number of Central graph of Ladder graph and Central graph of Complete graph denoted as $C\left(L_{n}\right)$ and $C\left(K_{n}\right)$ respectively. Also we discuss about the structural properties of $C\left(L_{n}\right)$ and $C\left(K_{n}\right)$.
\end{abstract}

Keywords: Central graph, b-colouringand b-chromatic number.

\section{INTRODUCTION AND PRELIMINARIES}

All graphs considered here are finite and simple. Notations and terminology not defined here will conform to those in (Bondy and Murty, 1976).

For a graph $G$, let $V(G), E(G), p(G), q(G)$ and

$\Delta(G), \delta(G)$, respectively, be the set of vertices, the set of edges, the order, the size, the maximum and minimum degree of $G$.

Let $G$ be a graph without loops and multiple edges with vertex set $V(G)$ and edge set $E(G)$. The smallest number $k$ for which $G$ admits a colouring with $k$ colours is the chromatic number $\chi(G)$ of $G$. Many graph invariants related to colourings have been defined. Most of them try to minimize the number of colours used to colour the vertices under some constraints. For some invariants, it is meaningful to try to maximize this number. The bchromatic number is one such example.

A b-colouring (Jakovac and Klavzar, 2010; Jakovac and Peterin, 2012; Kouider, 2002; Kouider and Zaker, 2006) of a graph $G$ is a proper colouring of the vertices of $G$ such that there exist a vertex in each colour class joined to at least a vertex in each other colour class; such a vertex is called a dominating vertex. The $b$-chromatic number of a graph $G$, denoted by $\varphi(G)$, is the maximal integer $k$ such that $G$ may have a b-colouring by $k$ colours. This parameter has been derived by Irving and Manlove in the year 1999.

The central graph (Thilagavathi et al., 2010; VernoldVivin et al., 2008; Vijayalakshmi and Thilagavathi, 2010) of any graph G is obtained by subdividing each edge of $G$ exactly once and joining all the non adjacentvertices of $\mathrm{G}$. By the definition $p C(G)=p+q$. For any $(p, q)$ graph there exactly $p$ vertices of degree $p-1$ and q vertices of degree 2 in $C$ (G).

\section{THE B-CHROMATIC NUMBER OFCENTRAL GRAPH OF LADDER GRAPH}

\author{
2.1. Theorem \\ For any integer $1<n<20, \varphi[C(L)]=n+n$
}

Proof

Let $L_{n}$ be any ladder with vertices [12] $v_{1}, v_{2} \ldots v_{n}$ labeled in anticlockwise direction. Let $v_{i j}$ be the newly introduced vertex in the edge connecting $v_{i}$ and $v_{j}, 1<i, j<2 n$ in $C\left(L_{n}\right)$. Now in $C\left(L_{n}\right)$, we see that each $v_{i}$ is adjacent with all the vertices except $v_{i+1}$ and $v_{2 n-(i-1)}$ for $i=1,2,3 \ldots 2 n$. Let $S=\left\{v_{i j} / 1<i, j<2 n\right\}$.

Now assign a proper colouring to these vertices as follows. Consider a colour class $C=\left\{c_{1}, c_{2}, c_{3} . . c_{n}\right\}$. For $i=1,2,3 \ldots 2 n$ assign the colour $c_{i}$ to the vertex $v_{i}$. Due to the above mentioned non adjacency this will not produce a b-chromatic colouring.

To overcome this, assign a proper colouring to $v_{i j}$ 's. consider an arbitrary vertex $v_{i}$, but $v_{i}$ is not adjacent with $v_{i+1}$ and $v_{i-1}$, thus the vertex $v_{i}$ to realize the colour $c_{i}$, we should colourv $v_{i, i+1}$ as $c_{i-1}$ and $v_{i, i-1}$ as $c_{i+1}$. Now $v_{i}$ will realize the colour $c_{i}$. Next consider the vertex $v_{i+1}$ which is coloured as $c_{i+1}$. In order to realize the colour $c_{i+1}$, colour the two neighbors of $v_{i+1}$ as $c_{i+1}$ and $c_{i}$ but by previous colouring $v_{i}$ had left out only one vertex to be coloured. Thus realization of $v_{i+1}$ is not possible. Proceeding in the same manner this will not be possible for remaining vertices. This implies that assigning different colours to $v_{i}$ is not possible otherwise there should be repetition of colours. A close examination will reveal that there should be minimum of ${ }^{n}$ repetitions.

Now assign the colour $c_{i-[i / 6]}$ to the vertex $v_{i}$ for $i=1,2,3, \ldots 2 n-1$ and assign the colour $c_{i\{\{[/ / G]+1\}}$ to the vertex $v_{2 n}$. 
To make the above colouring as bchromatic, assign a proper colouring to the remaining $v_{i j}{ }^{\prime}$. Suppose if we assign any new colour to any of the $v_{i j}$ 's as $c_{i-\{[i / 6]+1\}}$ it contradicts the definition of b-chromatic colouring. Hence we should assign only the existing colours to $v_{i j}$ 's inorder that all the vertices $v_{1}, v_{2} \ldots v_{2 n}$ realizes its own colour. Thus by the colouring procedure and under observation the above said colouring is maximal and b-chromatic colouring.

Example

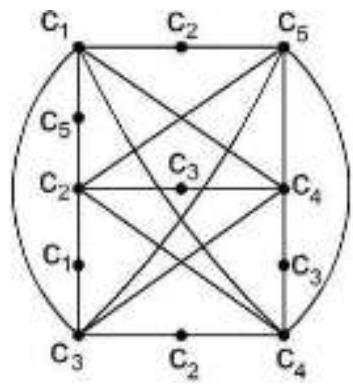

Figure: $1 \varphi\left[C\left(L_{3}\right)\right]=5$

2.2. Structural Properties of Central Graph ofLadder Graph

- $\quad$ Number of vertices in $C\left(L_{n}\right)=5 n-2$

- $\quad$ Maximum degree in $C\left(L_{n}\right)$ i.e. $\Delta=2 n-1$

- $\quad$ Minimum degree in $C\left(L_{n}\right)$ i.e. $\delta=2$

\section{B-CHROMATIC NUMBER OF CENTRALGRAPH OF COMPLETE GRAPH}

\subsection{Theorem}

For any integer $n>3, \varphi\left[C\left(K_{n}\right)\right]=n-1$

Example

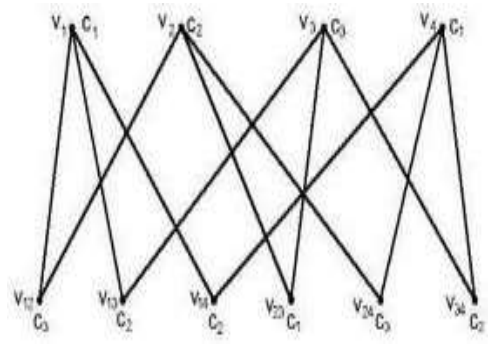

Figure :2 $\varphi\left[C\left(K_{4}\right)\right]=3$

3.2. Structural Properties of Central graph of Complete Graph

- $\quad$ Number of vertices in $C\left(K_{n}\right)=\frac{n(n+1)}{2}$
- $\quad$ Number of edges in $C\left(K_{n}\right)=n(n-1)$

- Maximum degree in $C\left(K_{n}\right)=(n-1)$

- $\quad$ Minimum degree in $C\left(K_{n}\right)=2$

- $\quad n$ vertices with maximum degree $n-1$

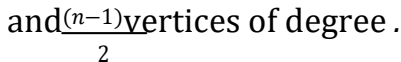

\section{REFERENCES}

Bondy, J.A. and U.S.R. Murty. (1976), Graph Theory with Applications, North-Holland, Amsterdam.

Irving R.W and D.F. Manlove (1999). The bchromatic number of a graph, Discrete. Appl.Math. 91:127-141.

Jakovac, M. and S. Klavzar, (2010). The b-chromatic number of cubic graphs, Graphs Combin. 26: 107-118.

Jakovac, M and I. Peterin, (2012). On the bchromatic number of some products, Studia Sci. Math. Hungar. 49: 156-169.

Kouider, M. b-chromatic number of a graph, subgraphs and degrees, Rapport interne LRI 1392.

Kouider, M. and A. El Sahili. About b-colouring of regular graphs, Rapport de Recherche No 1432, CNRS-Universite Paris Sud-LRI.

Kouider, M. and M. Maheo (2002). Some bounds for the b-chromatic number of a graph, Discrete Math. 256: 267-277.

Kouider, M. and M. Zaker. (2006) Bounds for the bchromatic number of some families of graphs, Discrete Math. 306: 617-623.

Thilagavathi K., D. Nidha and N. Roopesh. (2009). On harmonious coloring of $\mathrm{C}(\mathrm{Wn})$ and $\mathrm{C}(\mathrm{Fm}, \mathrm{n})$, Electronics Notes in Discrete mathematics. 33: 95-99.

Vernold Vivin .J, Akbar Ali.M. M and K Thilagavathi. (2008), On harmonious coloring of Central graph, Advances and Applications in DiscreteMathemetics. 2:17-33.

Vijayalakshmi, D.and K.Thilagavathi (2010). bcolouring of Central graphs, International Journal of Computer Applications, 3(11): 09758887.

Weisstei, M and Eric W."Ladder Graph" from MathWorld, Wikipedia, the free Encyclopedia. 